AP: Before you had your treatment, what did you think it would be like?

SP: I knew other people who had been given chemotherapy. They had felt crook all the time and had problems with vomiting. I knew it would not be pleasant, however I coped well with chemotherapy.

I did not know what I was treated with, but my treatment was for three days every three weeks. This was repeated six times. I had blood tests before each treatment and I had to stay in hospital for two nights each time.

AP: What adverse effects did you have?

SP: The doctors and nurses were very good - they told me what side effects to expect and what I could do about them. I only vomited twice, but all my hair fell out after the second treatment. I think I coped well. Apart from a couple of days when I overdid things, I did not feel too unwell.
AP: Were you relieved when the treatment was completed?

SP: I hoped the treatment was curing the cancer but I was pleased when the chemotherapy was over. I felt more lively and my hair grew back.

It is now nearly two years since I finished treatment. I am still playing golf and a few sets of tennis.

AP: Would you make the same choice if you had to make the decision about being treated again?

SP: I would have chemotherapy again if the doctors advised it.

AP: Do you have any advice to help elderly people with cancer decide about having or continuing treatment?

SP: It would be a bit rough to say that old people with cancer should not be treated. I think if you are reasonably fit you should go for the treatment. If you are advised to have chemotherapy, think positively and go for it.

\title{
Dental implications
}

\section{Prepared by Associate Professor R.G. Woods of the Australian Dental Association}

\section{Chemotherapy for elderly patients with advanced cancer: is it worth it? (page 80)}

The oral adverse effects of chemotherapy may complicate dental treatment in patients with cancer. ${ }^{1}$ Systemic adverse effects, for instance immunosuppression, are likely to complicate endodontic treatment or surgery. Taking a pretreatment medical history is essential to obtain information about the drugs being used to treat the cancer. You should also ask if the patient has had radiotherapy, particularly in the region of the head and neck.

Dental treatment needs to be planned with care. The risks of post-operative infection and delayed wound healing should be minimised. This could include antibiotic surgical prophylaxis. An increased risk of infection from periodontal disease and its treatment should be anticipated. Stomatitis may occur with or without oral ulceration and may be relieved by topical steroid therapy (triamcinolone).

Where possible, a comprehensive oral and dental examination should be made and any infection treated before chemotherapy begins. Following chemotherapy provision should be made for dentate patients, to have a regular preventive dentistry program to minimise the need for invasive dental treatment. Dental management of any patient, young or old, can be complicated by chemotherapy. Where there is any doubt concerning the effects of medication used or precautions needed, dentists should discuss the case with the patient's oncologist.

REFERENCE

1. Bonaventura A.Complications of cytotoxic therapy. Aust Prescr 1995;18:68.

\section{International success}

In June Dr John Dowden, the Editor of Australian Prescriber, received an outstanding service award from the Drug Information Association (DIA). The presentation ceremony took place at this year's annual meeting of the DIA in California. Dr Dowden accepted the award in front of an audience of approximately 7000 people in the San Diego Convention Centre.

While the award is primarily for Dr Dowden's work with the South-west Asia-Pacific Steering Committee of the DIA, it reflects well on Australian Prescriber. The journal is an important source of independent drug information in Australasia. The Executive Editorial Board congratulates the Editor on his award.

For more information about the DIA follow the Links from the Australian Prescriber web site at www.australianprescriber.com 